\section{Consideration of the sequence rule in rule P-94.2}

\section{by Hiroshi Izumi}

The optimization of substituents for drug design requires a comparison of the maximal common substructures (MCSs) of the lead candidates; the optimization of three-dimensional (3D) structures, in particular, has attracted the attention of pharmaceutical scientists [13]. For this reason, there is an increased need for methods to compare conformations other than superimposition $[4,5]$. To compare the conformations of the MCSs of different organic molecules, the four-atom selections for dihedral angle determination must correspond with each other. Rule P-94.2 of the International Union of Pure and Applied Chemistry (IUPAC) rules for the Nomenclature of Organic Chemistry [6] makes use of the sequence rule (P-92). Rule P-94.2 can be applied to identical molecules. However, with very few exceptions, Rule P-94.2 can also be used to compare conformations between MCSs of different molecules. I believe that a simple fusion of the selection criteria in Rule P-94.2 should be applied with very few exceptions.

\section{Rule P-94.2}

Rule P-94.2 of the IUPAC Rules for Nomenclature of Organic Chemistry [6] contains the following criteria to select an atom or group to define the torsion angle: a. If all the atoms or groups of a set are different, [select] that one [atom or group] of each set that has priority by the sequence rule;

b. If one [atom or group] of a set is unique, [select] that one;

c. If all [atoms or groups] of a set are identical, [select] that one which provides the smallest torsion angle.

Instead of these criteria, I propose a simple fusion of (a) and (b) in Rule P-94.2:

(a) If all the atoms or groups of a set are different, or if one atom or group is unique, select the atom or group that has priority by the sequence rule, except where one atom or group is unique between the rankings of each atom in the $n^{\text {th }}$ sphere and in the $(n+1)^{\text {th }}$ sphere (P-92.1.5 Exploration of a hierarchical digraph), in which case select that one;

(b) if all the atoms or groups of a set are identical, select the atom or group that provides the smallest torsion angle.

\section{Glutathione vs ophthalmic acid}

In the design of drugs, there is a strong need to compare the conformations of peptides and proteins [5]. According to Rule P-94.2, the conformations of main chains containing cysteine cannot be compared with chains containing other amino acids. For example, although the sulfanylmethyl $\left(\mathrm{HSCH}_{2}^{-}\right)$group is

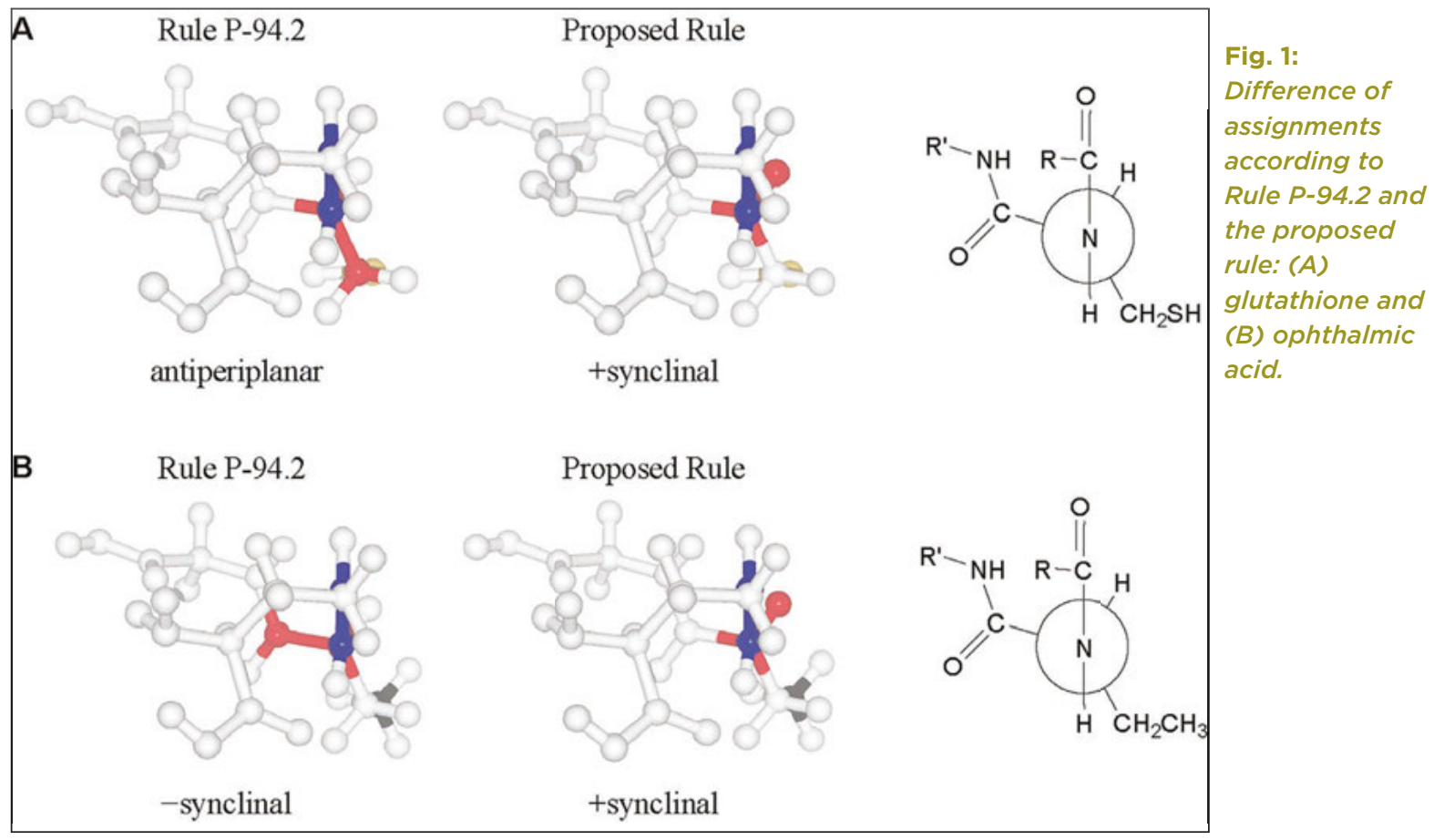


selected as the priority group of glutathione according to Rule P-94.2, the CONH group of ophthalmic acid or other amino acids has priority (Fig. 1). As a result, the assignments of conformations between glutathione and ophthalmic acid are different according to Rule P-94.2. In contrast, the methine $(\mathrm{CH})$ groups are selected according to the proposed rule in both cases, and the assignments of conformations become the same "+synclinal," because the highest priority atom is selected in the order closest to the center bond for dihedral angle determination.

\section{Cholesteryl pelargonate vs cyclohexyl nonanoate}

A case is troublesome if the selection of the priority group according to Rule P-94.2 is determined by a group at a distant location. Although the methine $(\mathrm{CH})$ group of cyclohexyl nonanoate, which has a highly symmetric structure, is selected as the priority group according to Rule P-94.2 (b), the methylene $\left(\mathrm{CH}_{2}\right)$ group of cholesteryl pelargonate, which is close to the alkene, has priority (Fig. 2). In contrast, the methine $(\mathrm{CH})$ groups are selected according to the proposed rule in both cases, and the assignments of conformations become the same "+synclinal", because the highest priority atom is selected for dihedral angle determination on the basis that it is closest to the center bond. In this way, the proposed rule can dramatically enhance the versatility of conformational comparisons.

\section{Acknowledgments}

This work was partly supported by JSPS KAKENHI Grant Number JP16K05711. The author thanks Dr. Rina K. Dukor, Professor Laurence A. Nafie, and Professor Hiroshi Ogino for discussions of this document. The author also thanks native-English-speaking professional editors from ELSS, Inc. for English proofreading.

Hiroshi Izumi < izumi.h@aist.go.jp> can be reached at the National Institute of Advanced Industrial Science and Technology (AIST), AIST Tsukuba West, 16-1 Onogawa, Tsukuba, Ibaraki 305-8569, Japan

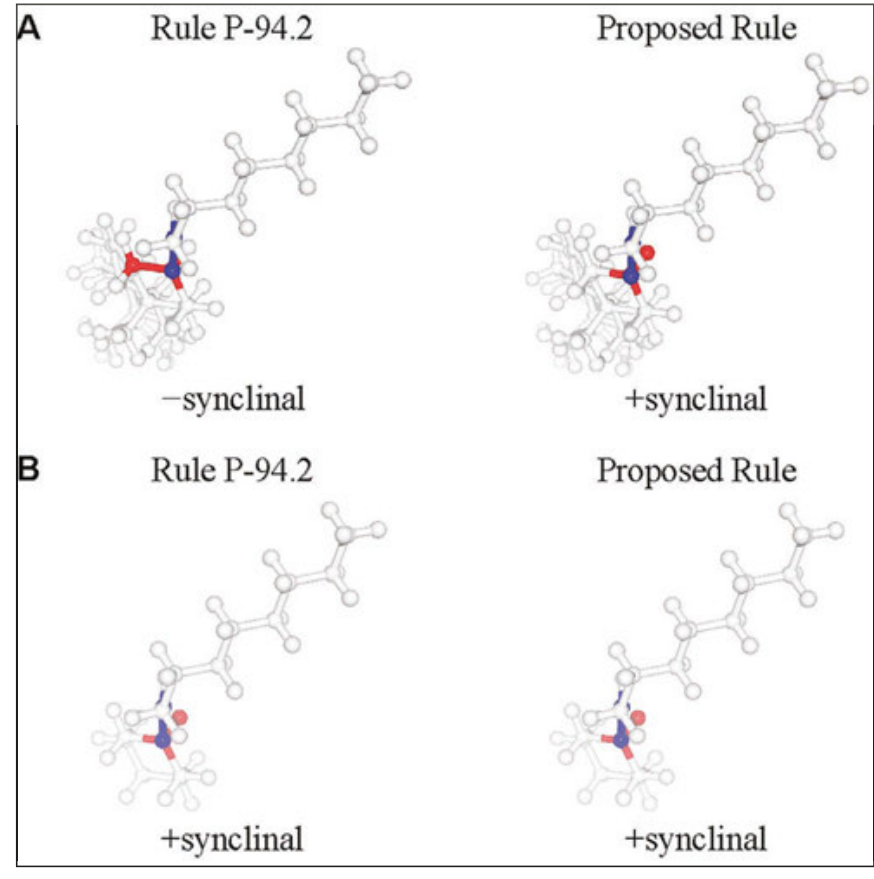

Fig. 2: Difference of assignments according to Rule P-94.2 and the proposed rule: (A) cholesteryl pelargonate and $(B)$ cyclohexyl nonanoate.

\section{References}

1. Z. Y. Xiao, S. L. Morris-Natschke, K. H. Lee. Med. Res. Rev. 36, 32 (2016).

2. C. E. Tinberg, S. D. Khare, J. Y. Dou, L. Doyle, J. W. Nelson, A. Schena, W. Jankowski, C. G. Kalodimos, K. Johnsson, B. L. Stoddard, D. Baker. Nature 501, 212 (2013).

3. W. L. Jorgensen. Acc. Chem. Res. 42, 724 (2009).

4. H. Izumi, L. A. Nafie, R. K. Dukor. Chirality 28, 370 (2016).

5. H. Izumi, A. Wakisaka, L. A. Nafie, R. K. Dukor. J. Chem. Inf. Model. 53, 584 (2013).

6. IUPAC Recommendations and Preferred Name 2013. Nomenclature of Organic Chemistry (the 'Blue Book'). Prepared for publication by H. A. Favre and W. H. Powell, The Royal Society of Chemistry, Cambridge (2013). 